\title{
Pregnancy outcome after intracytoplasmic sperm injection with strontium oocyte activation in a globozoospermic patient
}

\author{
Xiao-Yu Yang ${ }^{1, *}$, Jing Wang ${ }^{1, *}$, Jia-Yin Liu $^{1}$, Yan Gao ${ }^{1}$, Zuo-Min Zhou ${ }^{2}$, Jia-Hao Sha ${ }^{2}$, Wei Zhang ${ }^{3}$, Yu-Gui Cui ${ }^{1}$ \\ and Xiao-Qiao Qian ${ }^{1}$
}

Asian Journal of Andrology (2012) 14, 341-343; doi:10.1038/aja.2011.134; published online 9 January 2012

Dear Editor,

I am Dr Xiao-Yu Yang, from the Center of Clinical Reproductive Medicine in the First Affiliated Hospital at the NanJing Medical University, Nanjing, China. We present here a case report of a globozoospermic patient whose partner became pregnant after intracytoplasmic sperm injection (ICSI) with assisted oocyte activation (AOA). Globozoospermia is characterized by the presence of $100 \%$ roundheaded spermatozoa lacking an acrosome. The lack of the acrosome, which renders spermatozoa unable to bind to the zona pellucida or fuse with the oocyte oolemma, is considered to be the cause of infertility in these patients. ${ }^{1}$

The introduction of ICSI provided a possible solution for patients suffering from globozoospermia. Since then, many cases of successful pregnancies after ICSI with globozoospermic sperm have been reported; $;^{2,3}$ however, the fertilisation rate after ICSI in these cases has been low, and some patients failed ICSI treatment because of limited or failed fertilisation. ${ }^{1}$ Fertilisation failure was initially attributed to the reduced ability of globozoospermic sperm to activate the oocyte.

Rybouchkin et al. ${ }^{4}$ demonstrated that the fertilisation rate was improved by the addition of a calcium ionophore. After that, ICSI combined with AOA was effectively used in many studies to improve the fertilisation rate and embryo development and to achieve live births from globozoospermic sperm from men with previously failed fertilisation attempts. Various methods of AOA, including the use of a calcium ionophore and electrical and mechanical stimuli, have been reported to be effective for fertilisation in men with globozoospermia.,

Strontium treatment, which is proven to induce calcium oscillations in mice, was found to be an effective method for AOA in cases of low or absent fertilisation after ICSI. ${ }^{5-7}$ Whether strontium is effective at improving fertilisation by globozoospermic sperm has not been reported. We report a successful case of a twin pregnancy resulting in live births following ICSI and strontium oocyte activation with round-headed sperm.
A couple with primary infertility of 5 years' duration visited our centre. Both members of the couple were 27 years old and healthy, with no physical problems other than the husband's semen parameters. The wife had no fertility problems. Analysis of a semen sample showed normal values for volume $(3.3 \mathrm{ml})$, sperm concentration $\left(56 \times 10^{6} \mathrm{ml}^{-1}\right)$ and motility (45\%). The Diff-Quik staining method revealed that $100 \%$ of the spermatozoa were round-headed and lacking an acrosome ${ }^{8}$ (Figure 1). Assessment of the acrosome with fluorescent Pisum sativum agglutinin staining of the human spermatozoa was also conducted ${ }^{9}$ (Figure 1). Ultrastructural characteristics of the round-headed sperm were evaluated using a scanning electron microscope (JEOL Technics Ltd, Akishima-Shi, Tokyo, Japan) ${ }^{3}$ (Figure 1). Spherical heads without acrosomes are shown. The karyotypes of the couple were obtained from peripheral lymphocytes and were $46, \mathrm{XX}$ in the wife and 46,XY in the husband.

A conventional long protocol was used to stimulate follicular development. Two cycle attempts were conducted. In the first cycle, the couple decided to undergo the conventional ICSI procedure. ICSI was performed on 20 metaphase II oocytes. Following ICSI, four oocytes were normally fertilized. Three days after insemination, two embryos reached the four-cell stage and one reached the three-cell stage without fragmentation; one did not cleave. Two embryos in the four-cell stage were transferred; however, pregnancy did not occur.

After the failure of the first ICSI cycle, the couple decided to conduct $\mathrm{SrCl}_{2}$ oocyte activation on the retrieved oocytes as part of the next attempt. Only three reports involving four patients undergoing $\mathrm{SrCl}_{2}$ for human oocyte activation have been published, making $\mathrm{SrCl}_{2}$ oocyte activation highly experimental. The risks of the treatment were explained in detail, and informed consent was obtained from the couple. The study protocol was approved by the local ethics committee of the First Affiliated Hospital of NanJing Medical University.

The second attempt was conducted 10 months after the first one using the same long ovarian stimulation protocol. Fifteen metaphase II oocytes were obtained and were randomly divided into two groups. Ten oocytes (Group B) underwent ICSI treatment combined with

${ }^{1}$ State Key Laboratory of Reproductive Medicine, Center of Clinical Reproductive Medicine, First Affiliated Hospital, Nanjing Medical University, Nanjing 210029 , China; ${ }^{2}$ State Key Laboratory of Reproductive Medicine, Department of Histology and Embryology, Nanjing Medical University, Nanjing 210029 , China and ${ }^{3}$ Department of Urology, First Affiliated Hospital, Nanjing Medical University, Nanjing 210029, China

* These authors contributed equally to this work.

Correspondence: Dr XQ Qian (qian_xiaoqiao@yahoo.com.cn)

Received: 7 August 2011; Revised: 5 September 2011; Accepted: 19 September 2011; Published online: 9 January 2012 

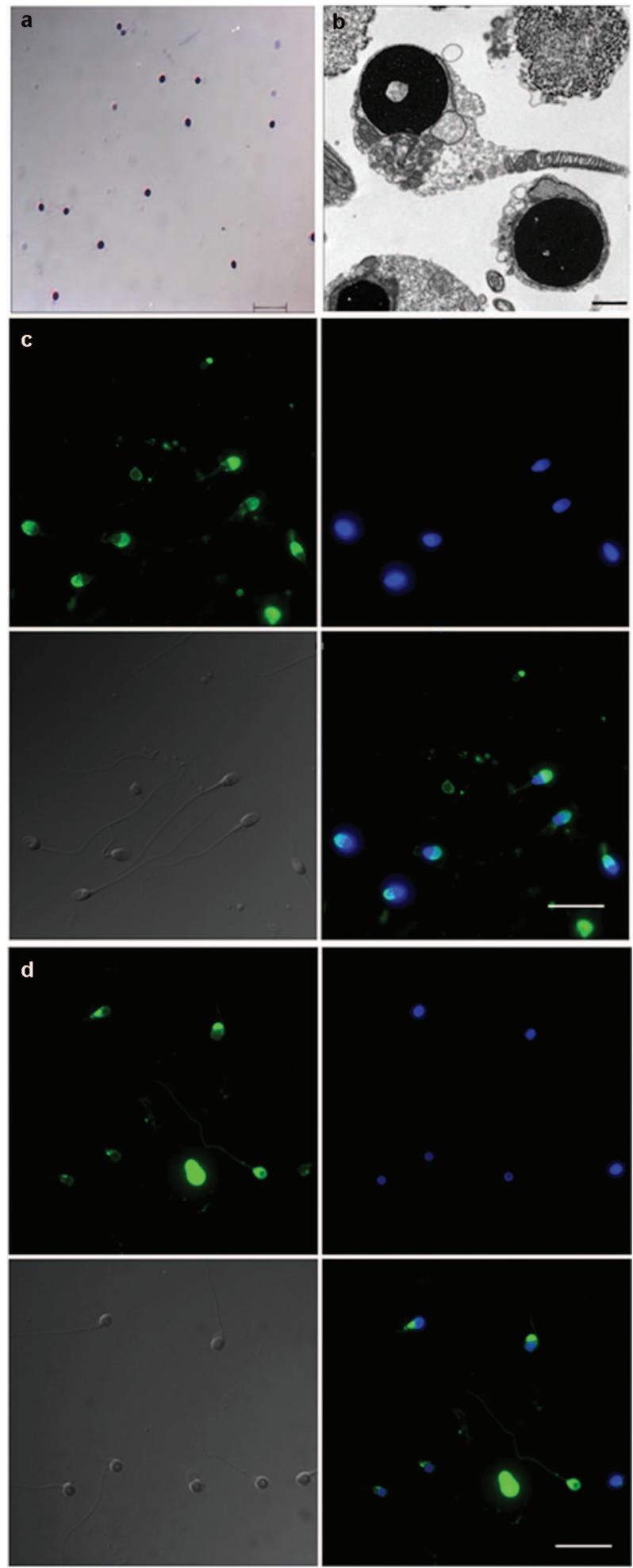

Figure 1 Round-headed sperm morphology in the globozoospermic patient. (a) Round-headed sperm morphology under a light microscope (scale bar=10 $\mu \mathrm{m}$ ); (b) scanning electron micrograph of round-headed spermatozoa (scale bar $=1 \mu \mathrm{m}$ ); (c) normal human spermatozoa fluorescence image after PSA staining (scale bar $=10 \mu \mathrm{m}$ ); (d) round-headed spermatozoa fluorescence image after PSA staining (scale bar $=10 \mu \mathrm{m})$. PSA, Pisum sativum agglutinin.

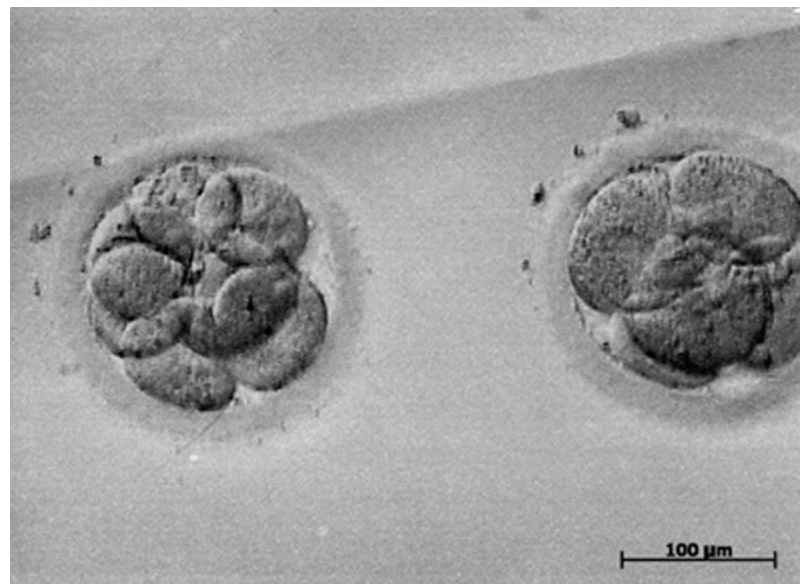

Figure 2 Image of day 3 embryos after $\mathrm{SrCl}_{2}$ activation under a light microscope (scale bar $=100 \mu \mathrm{m}$ ). The left embryo reached the eight-cell stage with no fragmentation; the right one reached the five-cell stage with $5 \%$ fragmentation.

$\mathrm{SrCl}_{2}$ oocyte activation, and the other five (Group A) underwent ICSI only. After ICSI, the oocytes of Group A were activated in calcium-free human tubal fluid medium (SAGE BioPharma, Bedminster, NJ, USA) containing $10 \mathrm{mmol} \mathrm{l}^{-1} \mathrm{SrCl}_{2}$ (Sigma-Aldrich, Madrid, Spain) for $10 \mathrm{~min}$ at $37^{\circ} \mathrm{C}$ and $5 \% \mathrm{CO}_{2}$. Following AOA, the oocytes were rinsed thoroughly in in vitro fertilisation medium (Medicult, Maaloev DK2760, Denmark) and transferred to P1 medium containing 10\% (v/v) serum substitute supplement and incubated at $37{ }^{\circ} \mathrm{C}$ under $5 \% \mathrm{CO}_{2}$.

One oocyte from Group A and two oocytes from Group B were normally fertilized. Three days after microinjection, the one embryo from Group A reached the four-cell stage with 5\% fragmentation, while one of the two embryos from Group B reached the eight-cell stage with no fragmentation, and the other one reached the five-cell stage with 5\% fragmentation. The two embryos (Figure 2) that underwent $\mathrm{SrCl}_{2}$ activation were transferred. Two healthy female infants without any congenital abnormalities were delivered at 37 weeks gestation by caesarean section. Table 1 shows the patient and cycle characteristics.

$\mathrm{AOA}$ is used to induce calcium oscillations in oocyte activation. AOA methods, such as the popular calcium ionophore method and the method of electrostimulation, mostly result in a single transient increase of intracellular calcium in the oocytes. ${ }^{1} \mathrm{SrCl}_{2}$, unlike most of the AOA methods, was reported to cause oocyte activation accompanied by $\mathrm{Ca}^{2+}$ oscillations in mice. ${ }^{7}$ Zhang et al. ${ }^{10}$ speculated that $\mathrm{Sr}^{2+}$ probably promotes $\mathrm{Ca}^{2+}$ oscillations in mouse oocytes and embryos by sensitising the IP3 receptors (the $\mathrm{Ca}^{2+}$ release channels)

Table 1. Characteristics of the two cycles with globozoospermic sperm. (female: 27 years; male: 27 years)

\begin{tabular}{lccc} 
& & \multicolumn{2}{c}{ Second Cycle } \\
\cline { 3 - 4 } SrCl $_{2}$ activation & & Group A & Group B \\
\hline No. of mature oocytes & 20 & 5 & 10 \\
No. of fertilised oocytes & 4 & 1 & 2 \\
Fertilisation rate (\%) & 20 & 20 & 20 \\
Cleavage rate (\%) & 75 & 100 & 100 \\
No. of embryos transferred & 2 & No & 2 \\
Clinical pregnancy & No & No & Yes \\
Implantation rate (\%) & No & No & 100 \\
Live birth & No & No & 2 (female) \\
\hline
\end{tabular}

${ }^{a}$ These oocytes were activated with $10 \mathrm{mmol} / \mathrm{L} \mathrm{SrCl}_{2}$ for 10 min after microinjection. 
to release $\mathrm{Ca}^{2+}$. The efficiency of $\mathrm{SrCl}_{2}$ for oocyte activation varies depending on the species. ${ }^{5}$ It is most effective in mice, but in human oocytes, calcium oscillation has not been observed, which is the key to oocyte activation. Four case reports have been published demonstrating improved fertilisation rates and embryo quality following strontium was used for AOA in humans with low or no fertility, and successful pregnancies and live births have been achieved. ${ }^{5-7}$

Our study is the first reported case of successful pregnancy and live births after strontium was used for AOA with globozoospermic sperm. However, the fertilisation rate was not improved after strontium treatment, even though it seemed to improve the quality of the embryos. We could not confirm whether the twin pregnancy benefited from the strontium activation after ICSI. The potential toxic effects of AOA with strontium have not been fully determined in clinical ICSI, and the safety of strontium stimulus remains to be assessed. ${ }^{6}$ Several studies have indicated that no physical or mental developmental disorders are associated with babies born after strontium stimulus, up to 12 months after birth. ${ }^{5-7}$ However, there have not been any reports of genetic analysis in humans born following strontium stimulus treatment. The long-term effect of the potential toxicity of strontium on humans remains unknown.

In conclusion, oocyte activation using strontium for fertilisation with round-headed sperm may not be effective in increasing the fertilisation rate, even though the only improvement we measured was in embryo quality.

\section{AUTHOR CONTRIBUTIONS}

XQQ, XYY, WZ, ZMZ and JHS conceived and designed the study. XQQ completed the embryo work in the laboratory. JYL and YG completed the clinical work. YGC revised the manuscript for important intellectual content. XYY and JW wrote the manuscript, which was read and approved by all authors.

\section{COMPETING FINANCIAL INTERESTS}

The authors declare no competing financial interests.

1 Dam AH, Feenstra I, Westphal JR, Ramos L, van Golde RJ et al. Globozoospermia revisited. Hum Reprod Update 2007; 13: 63-75.

2 Liu J, Nagy Z, Joris H, Tournaye H, Devroey P et al. Successful fertilization and establishment of pregnancies after intracytoplasmic sperm injection in patients with globozoospermia. Hum Reprod 1995; 10: 626-9.

3 Dirican EK, Isik A, Vicdan K, Sozen E, Suludere Z. Clinical pregnancies and livebirths achieved by intracytoplasmic injection of round headed acrosomeless spermatozoa with and without oocyte activation in familial globozoospermia: case report. Asian J Androl 2008; 10: 332-6.

4 Rybouchkin A, van der Elst J, de Sutter P, Dhont M. 'Globe-headed spermatozoa' and ICSI. Fertil Steril 1998; 69: 361-2.

5 Yanagida K, Morozumi K, Katayose H, Hayashi S, Sato A. Successful pregnancy after ICSI with strontium oocyte activation in low rates of fertilization. Reprod Biomed Online 2006; 136: 801-6.

6 Kyono K, Kumagai S, Nishinaka C, Nakajo Y, Uto H et al. Birth and follow-up of babies born following ICSI using $\mathrm{SrCl}_{2}$ oocyte activation. Reprod Biomed Online 2008; 17 $53-8$.

7 Chen J, Qian Y, Tan Y, Mima H. Successful pregnancy following oocyte activation by strontium in normozoospermic patients of unexplained infertility with fertilisation failures during previous intracytoplasmic sperm injection treatment. Reprod Fertil Dev 2010; 22: 852-5.

8 World Health Organization. WHO Manual for the Examination of Semen and Sperm/Cervical Mucus Interaction. Cambridge: Cambridge University Press 1999. p76.

9 Liu DY, Baker HW. The proportion of human sperm with poor morphology but norma intact acrosomes detected with Pisum sativum agglutinin correlates with fertilization in-vitro. Fertil Steril 1988; 50: 288-93.

10 Zhang D, Pan L, Yang LH, He XK, Huang XY et al. Strontium promotes calcium oscillations in mouse meiotic oocytes and early embryos through InsP3 receptors, and requires activation of phospholipase and the synergistic action of InsP3. Hum Reprod 2005; 20: 3053-6. 